\title{
EXPLICIT INVERSE OF THE PASCAL MATRIX PLUS ONE
}

\author{
SHENG-LIANG YANG AND ZHONG-KUI LIU
}

Received 5 June 2005; Revised 21 September 2005; Accepted 5 December 2005

This paper presents a simple approach to invert the matrix $P_{n}+I_{n}$ by applying the Euler polynomials and Bernoulli numbers, where $P_{n}$ is the Pascal matrix.

Copyright (c) 2006 Hindawi Publishing Corporation. All rights reserved.

\section{Introduction}

The Pascal matrix has been known since ancient times, and it arises in many different areas of mathematics. However, it has been studied carefully only recently, see [1,3-5]. For any integer $n>0$, the $n \times n$ Pascal matrix $P_{n}$ is defined with the binomial coefficients by

$$
P_{n}(i, j)= \begin{cases}\left(\begin{array}{l}
i-1 \\
j-1
\end{array}\right) & \text { if } i \geq j \geq 1 \\
0 & \text { otherwise. }\end{cases}
$$

It is known that the $n \times n$ inverse matrix $P_{n}^{-1}$ is given by

$$
P_{n}(i, j)= \begin{cases}(-1)^{i-j}\left(\begin{array}{l}
i-1 \\
j-1
\end{array}\right) & \text { if } i \geq j \geq 1, \\
0 & \text { otherwise. }\end{cases}
$$

The Hadamard product $A \circ B$ of two matrices is the matrix obtained by coordinatewise multiplication: $(A \circ B)(i, j)=A(i, j) B(i, j)$. Let $\Gamma_{n}$ be the $n \times n$ lower triangular matrices defined by

$$
\Gamma_{n}(i, j)= \begin{cases}(-1)^{i-j} & \text { if } i \geq j \geq 1 \\ 0 & \text { otherwise }\end{cases}
$$


2 Explicit inverse of the Pascal matrix plus one

then the inverse of the Pascal matrix can be represented as the Hadamard product $P_{n}^{-1}=$ $P_{n} \circ \Gamma_{n}$. For example, if $n=5$, then

$$
\begin{aligned}
P_{5} & =\left(\begin{array}{ccccc}
1 & 0 & 0 & 0 & 0 \\
1 & 1 & 0 & 0 & 0 \\
1 & 2 & 1 & 0 & 0 \\
1 & 3 & 3 & 1 & 0 \\
1 & 4 & 6 & 4 & 1
\end{array}\right), \\
P_{5}^{-1} & =\left(\begin{array}{ccccc}
1 & 0 & 0 & 0 & 0 \\
-1 & 1 & 0 & 0 & 0 \\
1 & -2 & 1 & 0 & 0 \\
-1 & 3 & -3 & 1 & 0 \\
1 & -4 & 6 & -4 & 1
\end{array}\right)=\left(\begin{array}{lllll}
1 & 0 & 0 & 0 & 0 \\
1 & 1 & 0 & 0 & 0 \\
1 & 2 & 1 & 0 & 0 \\
1 & 3 & 3 & 1 & 0 \\
1 & 4 & 6 & 4 & 1
\end{array}\right) \circ\left(\begin{array}{ccccc}
1 & 0 & 0 & 0 & 0 \\
-1 & 1 & 0 & 0 & 0 \\
1 & -1 & 1 & 0 & 0 \\
-1 & 1 & -1 & 1 & 0 \\
1 & -1 & 1 & -1 & 1
\end{array}\right) .
\end{aligned}
$$

Now we consider the sum of the Pascal matrix and the identity matrix $P_{n}+I_{n}$, where $I_{n}$ is the $n \times n$ identity matrix. We call $P_{n}+I_{n}$ the Pascal matrix plus one simply. An interesting fact is that the inverse of $P_{n}+I_{n}$ is related to $P_{n}$ closely. For instance,

$$
P_{6}+I_{6}=\left(\begin{array}{cccccc}
2 & 0 & 0 & 0 & 0 & 0 \\
1 & 2 & 0 & 0 & 0 & 0 \\
1 & 2 & 2 & 0 & 0 & 0 \\
1 & 3 & 3 & 2 & 0 & 0 \\
1 & 4 & 6 & 4 & 2 & 0 \\
1 & 5 & 10 & 10 & 5 & 2
\end{array}\right), \text { }\left(P_{6}+I_{6}\right)^{-1}=\left(\begin{array}{ccccccc}
\frac{1}{2} & 0 & 0 & 0 & 0 & 0 \\
-\frac{1}{4} & \frac{1}{2} & 0 & 0 & 0 & 0 \\
0 & -\frac{2}{4} & \frac{1}{2} & 0 & 0 & 0 \\
\frac{1}{8} & 0 & -\frac{3}{4} & \frac{1}{2} & 0 & 0 \\
0 & \frac{4}{8} & 0 & -\frac{4}{4} & \frac{1}{2} & 0 \\
-\frac{1}{4} & 0 & \frac{10}{8} & 0 & -\frac{5}{4} & \frac{1}{2}
\end{array}\right)
$$




$$
=\left(\begin{array}{llllll}
1 & 0 & 0 & 0 & 0 & 0 \\
1 & 1 & 0 & 0 & 0 & 0 \\
1 & 2 & 1 & 0 & 0 & 0 \\
1 & 3 & 3 & 1 & 0 & 0 \\
1 & 4 & 6 & 4 & 1 & 0 \\
1 & 5 & 10 & 10 & 5 & 1
\end{array}\right) \circ\left(\begin{array}{cccccc}
\frac{1}{2} & 0 & 0 & 0 & 0 & 0 \\
-\frac{1}{4} & \frac{1}{2} & 0 & 0 & 0 & 0 \\
0 & -\frac{1}{4} & \frac{1}{2} & 0 & 0 & 0 \\
\frac{1}{8} & 0 & -\frac{1}{4} & \frac{1}{2} & 0 & 0 \\
0 & \frac{1}{8} & 0 & -\frac{1}{4} & \frac{1}{2} & 0 \\
-\frac{1}{4} & 0 & \frac{1}{8} & 0 & -\frac{1}{4} & \frac{1}{2}
\end{array}\right) .
$$

This suggests that there may exist a sequence of constants $\left\{a_{n}\right\}_{n=0}^{\infty}$ such that $\left(P_{n}+\right.$ $\left.I_{n}\right)^{-1}=P_{n} \circ \Delta_{n}$, where the matrix $\Delta_{n}$ is a lower triangular matrix with generic element $\Delta_{n}(i, j)=a_{i-j}$ when $i \geq j$. Aggarwala and Lamoureux [2] have showed that these constants are values of the Dirichlet eta function evaluated at negative integers, or more generally, certain polylogarithm functions evaluated at the number -1 . In this note, we will give a new simple approach to invert the matrix $P_{n}+I_{n}$ by applying the Euler polynomials. As a result, we will show that these constants are values of the Euler polynomials evaluated at the number 0 .

The Euler polynomials $E_{n}(x)$ are defined by means of the following generating function (see [7]):

$$
\sum_{n=0}^{\infty} E_{n}(x) \frac{t^{n}}{n !}=\frac{2 e^{t x}}{e^{t}+1},
$$

since $\sum_{n=0}^{\infty}\left(E_{n}(x+1)+E_{n}(x)\right)\left(t^{n} / n !\right)=\sum_{n=0}^{\infty} E_{n}(x+1)\left(t^{n} / n !\right)+\sum_{n=0}^{\infty} E_{n}(x)\left(t^{n} / n !\right)=$ $2 e^{t(x+1)} /\left(e^{t}+1\right)+2 e^{t x} /\left(e^{t}+1\right)=2 e^{t x}=\sum_{n=0}^{\infty} 2 x^{n}\left(t^{n} / n !\right)$. Comparing the coefficients of $t^{n} / n !$ in this equation, we obtain

$$
E_{n}(x+1)+E_{n}(x)=2 x^{n}, \quad n \geq 0 .
$$

The following lemmas are well known and can be found in [9], we give a short proof for the sake of completeness.

Lemma 1.1. For all $n \geq 0$,

$$
\begin{gathered}
E_{n}(x+y)=\sum_{k=0}^{n}\left(\begin{array}{l}
n \\
k
\end{array}\right) E_{k}(x) y^{n-k}, \\
E_{n}(x+1)=\sum_{k=0}^{n}\left(\begin{array}{l}
n \\
k
\end{array}\right) E_{k}(x) .
\end{gathered}
$$

Proof. $\sum_{n=0}^{\infty} E_{n}(x+y)\left(t^{n} / n !\right)=2 e^{t(x+y)} /\left(e^{t}+1\right)=\left(2 e^{t x} /\left(e^{t}+1\right)\right) e^{t y}=\left(\sum_{n=0}^{\infty} E_{n}(x)\left(t^{n} /\right.\right.$ $n !))\left(\sum_{n=0}^{\infty} y^{n}\left(t^{n} / n !\right)\right)=\sum_{n=0}^{\infty}\left(\sum_{k=0}^{n}\left(\begin{array}{l}n \\ k\end{array}\right) E_{k}(x) y^{n-k}\right)\left(t^{n} / n !\right)$. Comparing the coefficients of $t^{n} / n$ ! in this equation, we obtain (1.8). In particular, when $y=1$, we get (1.9). 
4 Explicit inverse of the Pascal matrix plus one

From (1.7) and (1.9), we obtain

$$
\frac{1}{2} \sum_{k=0}^{n}\left(\begin{array}{l}
n \\
k
\end{array}\right) E_{k}(x)+\frac{1}{2} E_{n}(x)=x^{n}, \quad n \geq 0 .
$$

If we set $x=0$ in (1.8), we get $E_{n}(y)=\sum_{k=0}^{n}\left(\begin{array}{l}n \\ k\end{array}\right) E_{n-k}(0) y^{k}$, that is,

$$
E_{n}(x)=\sum_{k=0}^{n}\left(\begin{array}{l}
n \\
k
\end{array}\right) E_{n-k}(0) x^{k}, \quad n \geq 0 .
$$

Let $E(x)$ and $X(x)$ be the $n \times 1$ matrices defined by $E(x)=\left[E_{0}(x), E_{1}(x), \ldots, E_{n-1}(x)\right]^{T}$, $X(x)=\left[1, x, \ldots, x^{n-1}\right]^{T}$, and let $\bar{E}_{n}$ be $n \times n$ lower triangular matrices defined by

$$
\bar{E}_{n}(i, j)= \begin{cases}\left(\begin{array}{l}
i-1 \\
j-1
\end{array}\right) E_{i-j}(0) & \text { if } i \geq j \geq 1 \\
0 & \text { otherwise. }\end{cases}
$$

Then (1.10), (1.11) can be represented as matrix equations, respectively,

$$
\begin{gathered}
\frac{1}{2}\left(P_{n}+I_{n}\right) E(x)=X(x), \\
E(x)=\bar{E}_{n} X(x) .
\end{gathered}
$$

Thus, we have

$$
\begin{aligned}
& \left(P_{n}+I_{n}\right)^{-1} \\
& =\frac{1}{2} \bar{E}_{n}
\end{aligned}
$$

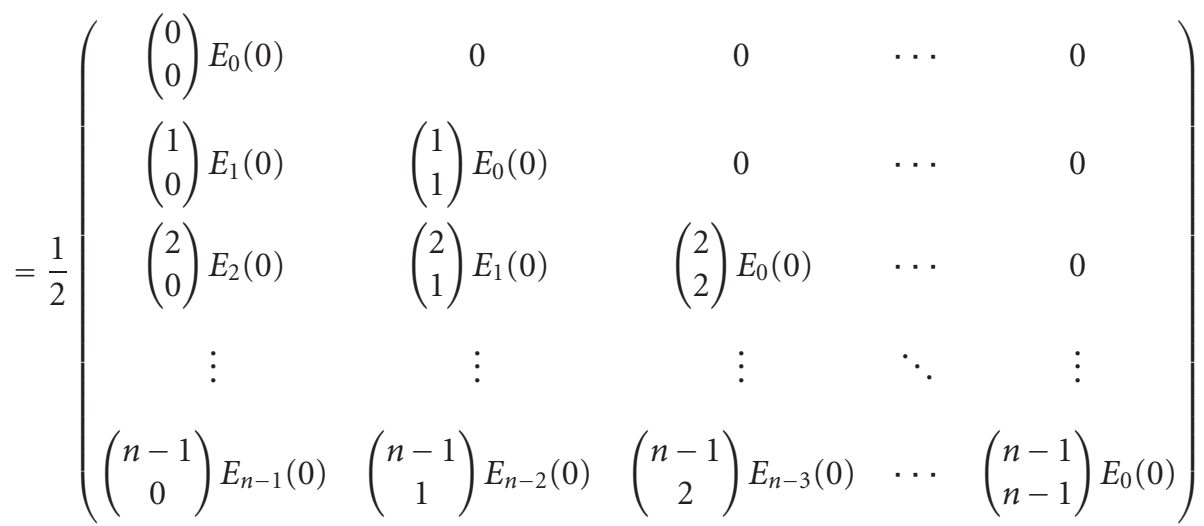


The Bernoulli numbers $B_{n}$ are defined by (see [7])

$$
\sum_{n=0}^{\infty} B_{n} \frac{t^{n}}{n !}=\frac{t}{e^{t}-1}
$$

It is known (see $[6,8])$ that the Euler polynomials can be expressed by the Bernoulli numbers as

$$
E_{n}(x)=\frac{1}{n+1} \sum_{k=1}^{n+1}\left(2-2^{k+1}\right)\left(\begin{array}{c}
n+1 \\
k
\end{array}\right) B_{k} x^{n+1-k}
$$

Putting $x=0$ in (1.16) gives

$$
E_{n}(0)=\frac{2\left(1-2^{n+1}\right) B_{n+1}}{n+1}
$$

for all integers $n \geq 0$. Therefore, we obtain an explicit inverse of the Pascal matrix plus one as follows.

Theorem 1.2. For $n \geq 1$, the $n \times n$ inverse matrix $Q_{n}=\left(P_{n}+I_{n}\right)^{-1}$ is given by

$$
Q_{n}(i, j)= \begin{cases}\frac{1}{2}\left(\begin{array}{l}
i-1 \\
j-1
\end{array}\right) E_{i-j}(0) & \text { if } i \geq j \geq 1, \\
0 & \text { if } i<j\end{cases}
$$

or

$$
Q_{n}(i, j)= \begin{cases}\left(\begin{array}{l}
i-1 \\
j-1
\end{array}\right) \frac{\left(1-2^{i-j+1}\right) B_{i-j+1}}{i-j+1} & \text { if } i \geq j \geq 1, \\
0 & \text { if } i<j .\end{cases}
$$

In view of the Hadamard product, the inverse matrix $\left(P_{n}+I_{n}\right)^{-1}$ is the Hadamard product of the Pascal matrix $P_{n}$ and the matrix $\Delta_{n}$, where $\Delta_{n}$ is the $n \times n$ lower triangular matrices defined by

$$
\Delta_{n}(i, j)= \begin{cases}\frac{1}{2} E_{i-j}(0) & \text { if } i \geq j \geq 1 \\ 0 & \text { if } i<j\end{cases}
$$

or

$$
\Delta_{n}(i, j)= \begin{cases}\frac{\left(1-2^{i-j+1}\right) B_{i-j+1}}{i-j+1} & \text { if } i \geq j \geq 1, \\ 0 & \text { if } i<j .\end{cases}
$$


6 Explicit inverse of the Pascal matrix plus one

The two functions, $\operatorname{Euler}(n, x)$ and $\operatorname{Bernoulli}(n)$, in the combinat library of the computer algebra system Maple are very useful in obtaining the matrix $Q_{n}$. For example, for $n=8$, we get

$$
Q_{8}=\left(P_{8}+I_{8}\right)^{-1}=\left(\begin{array}{cccccccc}
\frac{1}{2} & 0 & 0 & 0 & 0 & 0 & 0 & 0 \\
-\frac{1}{4} & \frac{1}{2} & 0 & 0 & 0 & 0 & 0 & 0 \\
0 & -\frac{1}{2} & \frac{1}{2} & 0 & 0 & 0 & 0 & 0 \\
\frac{1}{8} & 0 & -\frac{3}{4} & \frac{1}{2} & 0 & 0 & 0 & 0 \\
0 & \frac{1}{2} & 0 & -1 & \frac{1}{2} & 0 & 0 & 0 \\
-\frac{1}{4} & 0 & \frac{5}{4} & 0 & -\frac{5}{4} & \frac{1}{2} & 0 & 0 \\
0 & -\frac{3}{2} & 0 & \frac{5}{2} & 0 & -\frac{3}{2} & \frac{1}{2} & 0 \\
\frac{17}{16} & 0 & -\frac{21}{4} & 0 & \frac{35}{8} & 0 & -\frac{7}{4} & \frac{1}{2}
\end{array}\right) .
$$

Note that $Q_{n}(i, j)=0$ whenever $i<j$ or $i=j+2, j+4, j+6, \ldots$

\section{Acknowledgments}

This work is supported by Development Program for Outstanding Young Teachers in Lanzhou University of Technology and the NSF of Gansu Province of China. The authors wish to thank the referees for many valuable comments and suggestions that led to the improvement and revision of this note.

\section{References}

[1] L. Aceto and D. Trigiante, The matrices of Pascal and other greats, The American Mathematical Monthly 108 (2001), no. 3, 232-245.

[2] R. Aggarwala and M. P. Lamoureux, Inverting the Pascal matrix plus one, The American Mathematical Monthly 109 (2002), no. 4, 371-377.

[3] M. Bayat and H. Teimoori, Pascal K-eliminated functional matrix and its property, Linear Algebra and Its Applications 308 (2000), no. 1-3, 65-75.

[4] R. Brawer and M. Pirovino, The linear algebra of the Pascal matrix, Linear Algebra and Its Applications 174 (1992), 13-23.

[5] G. S. Call and D. J. Velleman, Pascal's matrices, The American Mathematical Monthly 100 (1993), no. $4,372-376$.

[6] G.-S. Cheon, A note on the Bernoulli and Euler polynomials, Applied Mathematics Letters 16 (2003), no. 3, 365-368.

[7] L. Comtet, Advanced Combinatorics. The Art of Finite and Infinite Expansions, D. Reidel, Dordrecht, 1974. 
[8] K. H. Rosen, J. G. Michaels, J. L. Gross, J. W. Grossman, and D. R. Shier (eds.), Handbook of Discrete and Combinatorial Mathematics, CRC Press, Florida, 2000.

[9] H. M. Srivastava and Á. Pintér, Remarks on some relationships between the Bernoulli and Euler polynomials, Applied Mathematics Letters 17 (2004), no. 4, 375-380.

Sheng-liang Yang: Department of Applied Mathematics, Lanzhou University of Technology, Lanzhou, Gansu 730050, China

E-mail address: slyang@lut.cn

Zhong-kui Liu: Department of Mathematics, Northwest Normal University, Lanzhou, Gansu 730070, China

E-mail address: liuzk@nwnu.edu.cn 


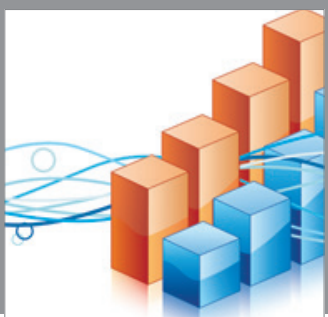

Advances in

Operations Research

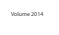

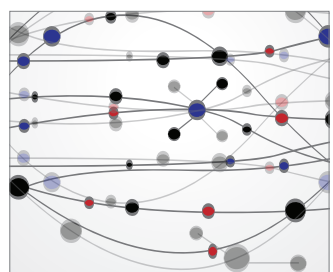

\section{The Scientific} World Journal
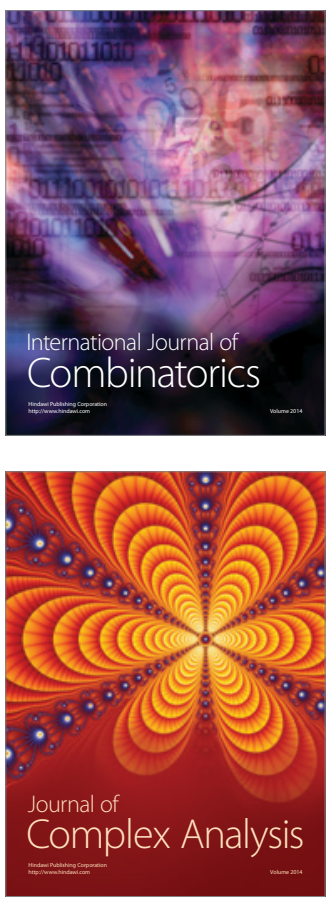

International Journal of

Mathematics and

Mathematical

Sciences
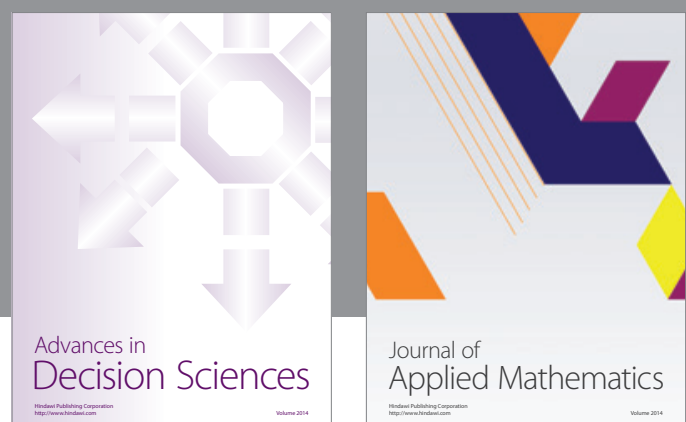

Journal of

Applied Mathematics
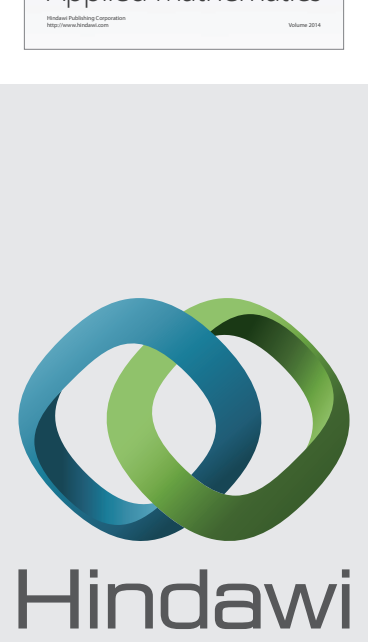

Submit your manuscripts at http://www.hindawi.com
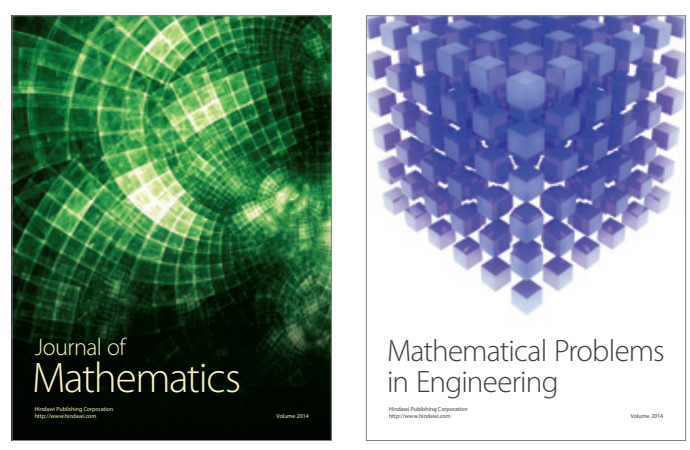

Mathematical Problems in Engineering
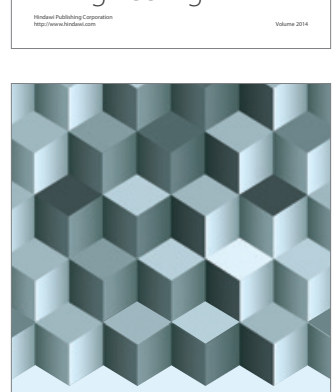

Journal of

Function Spaces
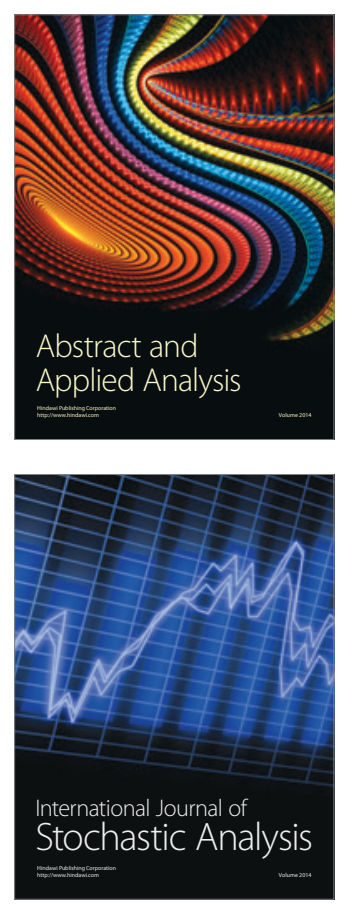

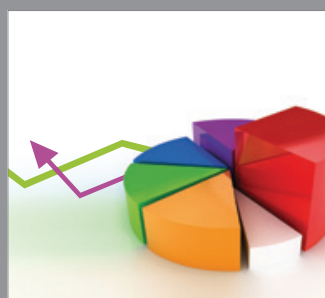

ournal of

Probability and Statistics

Promensencen
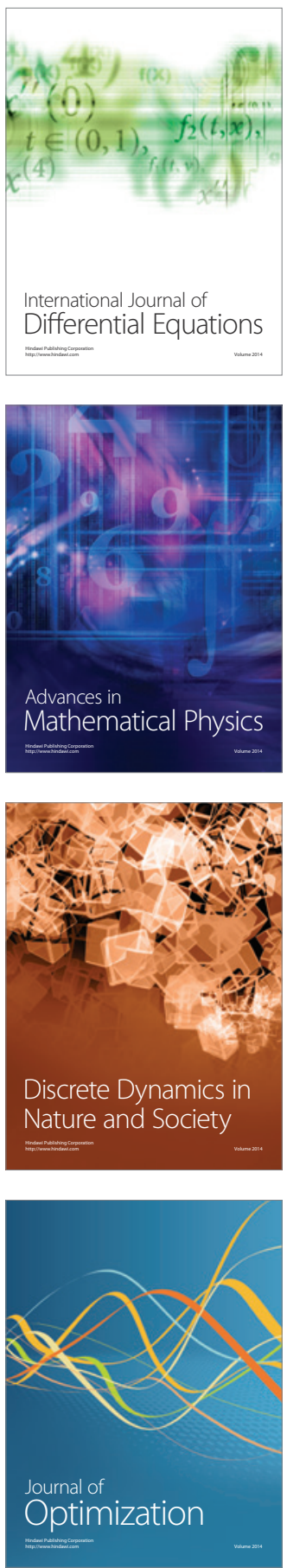